\title{
Impact of Human Leukocyte Antigen Polymorphisms in Human Immunodeficiency Virus Progression in a Paediatric Cohort Infected with a Mono-phyletic Human Immunodeficiency Virus-1 Strain
}

Carla Montesano ${ }^{1}$, Cesira Tiziana Bonanno ${ }^{2}$, Alba Grifoni', Caterina Di Sano ${ }^{3}$, Paolo Palma ${ }^{5}$, Guido Castelli-Gattinara ${ }^{5}$, Maurizio Mattei ${ }^{6}$, Giorgio Mancino ${ }^{7}$, Alfredo Salerno ${ }^{2}$, Vittorio Colizzi ${ }^{1}$ and Massimo Amicosante ${ }^{4,8^{*}}$

${ }^{1}$ Department of Biology, University of Rome, Tor Vergata, Rome, Italy

${ }^{2}$ Department of Biopathology and Biomedical Methodologies, University of Palermo, Palermo, Italy

3 Institute of Biomedicine and Molecular Immunology, National Research Council, Palermo, Italy

${ }^{4}$ Department of Biomedicine and Prevention, University of Rome, Tor Vergata, Rome, Italy

${ }^{5}$ Department of Immunology and Infectious Diseases, Bambino Gesù, Children's Hospital, Rome, Italy

${ }^{6}$ Animal Technology Station, University of Rome, Tor Vergata, Rome, Italy

${ }^{7}$ San Pietro Fatebenefratelli Hospital, Rome, Italy

${ }^{8}$ ProxAgen Ltd, Sofia, Bulgaria

\begin{abstract}
Objective: HLA polymorphisms within the peptide binding pocket have been associated with rapid and slowprogression to AIDS, suggesting that the capability to present efficiently HIV-1 epitopes is crucial for the infection control. To minimize the effects of genetic background due to population coming from different geographic area and viral strain variability in the cohort, an analysis of all the polymorphisms associated with the HLA-A, -B and -DR alleles has been performed in a cohort of children with a monophyletic HIV-1 infection (CRF02_AG) during an outbreak in Libya.
\end{abstract}

Methods: High-resolution HLA-typing has been performed in 58 children infected with a monophyletic strain of HIV-1: 26 Long-Term Non-Progressors (LTNP), 9 Slow-Progressors (SP) and 23 Fast-Progressors (FP). HLA amino acid polymorphism frequency has been compared in the in FP respect to LTNP.

Results: HLA-B resulted the most interesting locus of the study; 10 positions located in B-and F-pocket for peptidebinding have been found significant after Bonferroni's correction: 11S (LTNP=7.69\% FP=34.78\% OR=0.156 $P<0.05$ ), 74D (LTNP=15.38\%, FP=52.17\%, OR=0.167; $p<0.015)$ and 94T $(L T N P=15.38 \%, F P=52.17 \%, O R=0.045 ; p<0.001)$, resulted associated with AIDS progression; 66N (LTNP=42.31\% FP=8.7\% OR=7.7; $p<0.02), 80 \mathrm{I}(\mathrm{LTNP}=80.77 \%$, $\mathrm{FP}=34.78 \%, \quad \mathrm{OR}=7.86 ; \mathrm{p}<0.036), \quad 81 \mathrm{~A} \quad(\mathrm{LTNP}=84.61 \%, \quad \mathrm{FP}=47.83 \%, \quad \mathrm{OR}=6 ; \quad \mathrm{p}<0.015), \quad 82 \mathrm{~L} \quad(\mathrm{LTNP}=88.46 \%$, $F P=47.83 \%, O R=7.86 ; p<0.006)$ and $83 R(L T N P=88.46 \%, F P=47.83 \%, O R=7.86 ; p<0.006)$, has been associated with non-progression. Further, carrying Bw4-epitope resulted associated with LTNP (phenotype-frequency: LTNP $=88.46 \%$, $\mathrm{FP}=47.83 \%, \mathrm{OR}=8.36 ; \mathrm{p}<0.006)$, with homozygosis for $\mathrm{Bw} 4(\mathrm{LTNP}=30.8 \%, \mathrm{FP}=8.7 \%, \mathrm{p}<0.05)$ associated with delayed progression and homozygosis for Bw6 $(\mathrm{LTNP}=11.5 \%, \mathrm{FP}=52.1 \%, \mathrm{p}<0.05)$ associated with fast progression to AIDS.

Conclusion: The progression to AIDS might be in part determined by the binding capability of B-pocket and F-pocket of HLA-B and in part by the interaction of NK's inhibitory receptor with HLA-B Bw4-epitope which regulate innate immune response and might have important implications for a better disease control.

Keywords: Children; Epitope; HIV-1; HLA; Peptide binding motif; Susceptibility

\section{Introduction}

Susceptibility to HIV-1 infection, disease progression and clinical outcome are strongly influenced by differences observed in viral strains and host genetic factors [1].

Between a variety of genetic factors, certain specific alleles of human leukocyte antigen (HLA), both class I and II, have shown an effect on the outcome of HIV-1 infection, with many alleles found positively or negatively associated with disease progression to AIDS [1-3]. These findings are consistent with the role of HLA, particularly of class I molecules, in shaping the cell-mediated arm of anti-HIV immune response and in determining plasma viral load [4,5].

However, the HLA association studies with HIV disease progression and outcome have been limiting by multiple issues such as: the involvement of multiple HLA loci, the linkage disequilibrium, the diploid genome, the cohort effects, the different measures of outcome and the viral strain variability within the study subjects $[1,2]$.
Recently, the study of variation among the entire HLA molecules showed that the major genetic control of HIV progression is due to polymorphisms within the HLA binding groove [6,7].

In fact, apparently dissimilar HLA alleles may have similar antigen binding grooves, and thereby overlap in their capacity to present antigens [8]. The analysis of the single amino acid polymorphisms

*Corresponding author: Massimo Amicosante, Department of Biomedicine and Prevention, University of Rome, Tor Vergata, Via Montpellier 100133, Rome, Italy, Tel: +39-06-7259 6202; Fax: +39-06-7259 6202; E-mail: amicosan@uniroma2.it

Received December 21, 2013; Accepted January 29, 2014; Published February 11, 2014

Citation: Montesano C, Bonanno CT, Grifoni A, Di Sano C, Palma P, et al. (2014) Impact of Human Leukocyte Antigen Polymorphisms in Human Immunodeficiency Virus Progression in a Paediatric Cohort Infected with a Mono-phyletic Human Immunodeficiency Virus-1 Strain. J AIDS Clin Res 5: 282. doi:10.4172/21556113.1000282

Copyright: (c) 2014 Montesano C, et al. This is an open-access article distributed under the terms of the Creative Commons Attribution License, which permits unrestricted use, distribution, and reproduction in any medium, provided the original author and source are credited. 
might provide more relevant information on severity and diseases progression than the simply HLA allelic association.

The structure function studies indicate that the selection of antigenic peptides by the HLA receptors is dictated by the chemical-physical interaction between the amino acid side chains, lining receptor-like pockets on the floor of the HLA antigen binding groove, and the amino acid side chains of the antigenic peptide.

Therefore, in each subject the polymorphisms in HLA molecules could influence the capability to bind HIV-1 epitopes i.e. the phenotype of HIV-1 recognition, could be at the basis of the susceptibility to a more rapid progression to AIDS more than the carrying of a susceptible allele per se.

To test this hypothesis and to minimize the effects of (i) genetic background due to population coming from different or large geographic area and (ii) viral strain variability in the cohort, an analysis of all the polymorphisms associated with the HLA-A, -B and -DR alleles has been performed in a cohort of children infected with a monophyletic strain of HIV-1 (CRF02_AG) during an outbreak in the Benghazi Children Hospital in Libya [9].

\section{Materials and Methods}

\section{Study population}

The cohort involved in the outbreak of HIV infection at the "ElFath Children's Hospital" of Benghazi, includes 418 children, 18 mothers and 2 nurses. All the children resulted infected after attending the outpatients' service of the hospital or during hospitalization for at least once. The HIV infection and outbreak viral characterization has been confirmed as previously described [9], indicating in the cohort the presence of a defined cluster of HIV-1 clade A/G virus (CRF02_AG).

All the samples have been collected with the consent of children parents' and/or guardians. HLA-typing has been performed upon residual blood sample availability, after informed consent, from a subgroup of 114 children. They were 60 males and 54 females, all from the Libya Cyrenaica. This study population is representing a defined ethnic group geographical related with other populations from North Africa and Mediterranean areas as recently reported [10]. HCV coinfection was identified in 46 out of 114 children of the study population $[9,11]$.

The cohort subgroup evaluated in this study does not differ in its overall from the entire cohort for the mean value of $\mathrm{CD} 4$ cell counts, viral load, and frequency of subject in the different groups of classification for progression to AIDS. Thus, as far as the infection is concerned, the patients can be considered a homogeneous pool of subjects.

According to different definitions from the literature [12], these children have been divided in four groups. (1) Long Term Non Progressors (LTNP) includes patients with 6 or more years of infection, never on antiretroviral therapy (ARV), without any critical events;
(2) Slow Progressors (SP), patients with 6 or more years of infection with moderate clinical manifestation (CDC class A or B) or moderate immunosuppression (CDC class 2) without ARV; (3) Fast Progressors (FP), patients with severe clinical manifestation or immunosuppression before 6 years from infection, regardless the beginning of ARV and (4) Uncertain $(\mathrm{U})$, subjects on treatment for which therapy do not allowed an univocal classification. In order to clearly evaluate the association with HIV progression uncertain subjects have been excluded by this study.

\section{HLA typing and polymorphism assignment}

High Resolution HLA-A, -B and -DRB1 typing was performed through the ABI Prism 310 Genetic Analizer (Applied Biosystem, Foster City, CA, USA). Briefly, genomic DNA was obtained for each study subject by the salting-out method (Nuclear Laser Medicine, Italy) in according to protocol. Allele SEQR HLA-A and -B and allele SEQR HLA-DRB1 PCR/Sequencing kits (Abbott, Wiesbaden, Germany) have been used for HLA-A and -B or HLA-DRB1 typing respectively.

Supertype assignment of each HLA allele, i.e. based upon sharing the peptide binding motif, was performed accordingly the classification of HLA alleles in supertypes [13,14].

Polymorphism assignment has been performed translating every HLA alleles found in the cohort in the corresponding amino acid sequence considering only the polymorphic amino acid position obtained by protein sequence alignment in the IMGT/HLA database (http://www.ebi.ac.uk/imgt/hla/) as previously described [15]. Therefore, the phenotypic frequency of each polymorphic amino acid position has been evaluated between study groups.

Statistical analysis: All the data are expressed as frequency percentage. Comparison between frequencies is made by Chi-square, with Yates and Bonferroni's correction when appropriate. As previously reported [15]: (i) for HLA alleles' comparison, Bonferroni's correction has been performed using the total numbers of HLA alleles found in the study; (ii) for HLA supertype's comparison, Bonferroni's correction has been performed considering the total number of HLA supertypes found in the study; and (iii) for HLA amino acid polymorphisms Bonferroni's correction has been performed considering, within each position, the number of polymorphic amino acid residues found.

When in the comparisons the number of observations was below 5 a Chi-square per trend has been used. The Odds Ratio has been calculated as the ratio of the HLA polymorphisms frequency on FP respect to LTNP. GraphPad Prism version 5.0 has been used for all statistical analyses and graphs.

\section{Results}

Study groups characteristics are summarised in Table 1.

In the study population have been identified 30 alleles in HLA-A locus, 37 alleles in HLA-B locus and 31 alleles in HLA-DR locus (see

\begin{tabular}{|c|c|c|c|c|c|c|}
\hline & Patients & Sex (\% male $)$ & $\begin{array}{l}\text { Median age at the first } \\
\text { observation }\left(I Q R^{a}\right)\end{array}$ & $\begin{array}{l}\text { Median Nadir \%CD4+ } \\
\left(\mathrm{IQR}^{\mathrm{a}}\right)\end{array}$ & $\begin{array}{l}\text { HIV viral load Median } \\
\text { (range) }\end{array}$ & $\% \mathrm{ARV}^{\mathrm{b}}$ \\
\hline Long Term Non-Progressor & $26(22.8 \%)$ & $23.1 \%$ & $5.42(1.50-10.29)$ & $28.5 \%(25.0-32.0)$ & $400(50-2500)$ & 0.0 \\
\hline Slow Progressor & $9(7.9 \%)$ & $88.9 \%$ & $5.51(4.51-7.20)$ & $23.0 \%(19-25)$ & $2300(50-30000)$ & 0.0 \\
\hline Fast Progressor & $23(20.2 \%)$ & $60.9 \%$ & $3.97(2.10-6.68)$ & $13 \%(9-17)$ & $73000(5280-500000)$ & 68.2 \\
\hline Uncertain & $56(49.1 \%)$ & $57.1 \%$ & $1.79(1.32-28.88)$ & $21.5 \%(17-34)$ & $14500(400-270000)$ & 100.0 \\
\hline
\end{tabular}

aQR Inter-quartile range.

b\% of patients which started antiretroviral therapy.

Table 1: Demographic, immunological and therapeutic characteristics of the study groups classified according clinical progression. 
Citation: Montesano C, Bonanno CT, Grifoni A, Di Sano C, Palma P, et al. (2014) Impact of Human Leukocyte Antigen Polymorphisms in Human Immunodeficiency Virus Progression in a Paediatric Cohort Infected with a Mono-phyletic Human Immunodeficiency Virus-1 Strain. J AIDS Clin Res 5: 282. doi:10.4172/2155-6113.1000282

Page 3 of 6

Supplementary information S1 and S2). In its overall, the population resulted in Hardy-Weinberg equilibrium for all the HLA loci evaluated.

None of the HLA alleles and supertypes resulted associated after Bonferroni's correction with being LTNP or FP (see Supplementary information S1 and S2).

To assess the role of single amino acid positions in HIV progression, HLA alleles have been translated into the different amino acid position polymorphisms (Figure 1).

In HLA-A locus, 65 amino acid polymorphic positions have been evaluated. Figure 1A shows all the amino acid positions evaluated in our study and the location of the amino acid significant positions 76
$(\mathrm{P}=0.032), 77(\mathrm{P}=0.023)$ and $89(\mathrm{P}=0.028)$ within the HLA-A peptide binding pocket.

However, none of the 144 HLA-A polymorphisms evaluated showed a significant association with being LTNP or FP after Bonferroni's correction (Table 2 and S3).

In HLA-B locus, among the 49 amino acid polymorphic positions studied, 10 positions located in $\mathrm{B}$ and $\mathrm{F}$ peptide binding pocket have been found significant after Bonferroni's correction (Figure 1B).

Specifically, B-pocket related amino acid positions $24(\mathrm{P}=0.016)$, $45(\mathrm{P}=0.018), 62(\mathrm{P}=0.012), 65(\mathrm{P}=0.006), 66(\mathrm{P}=0.006)$, and $\mathrm{F}-$ pocket related amino acid positions $11(\mathrm{P}=0.016), 74(\mathrm{P}=0.006), 77(\mathrm{P}=0.013)$,

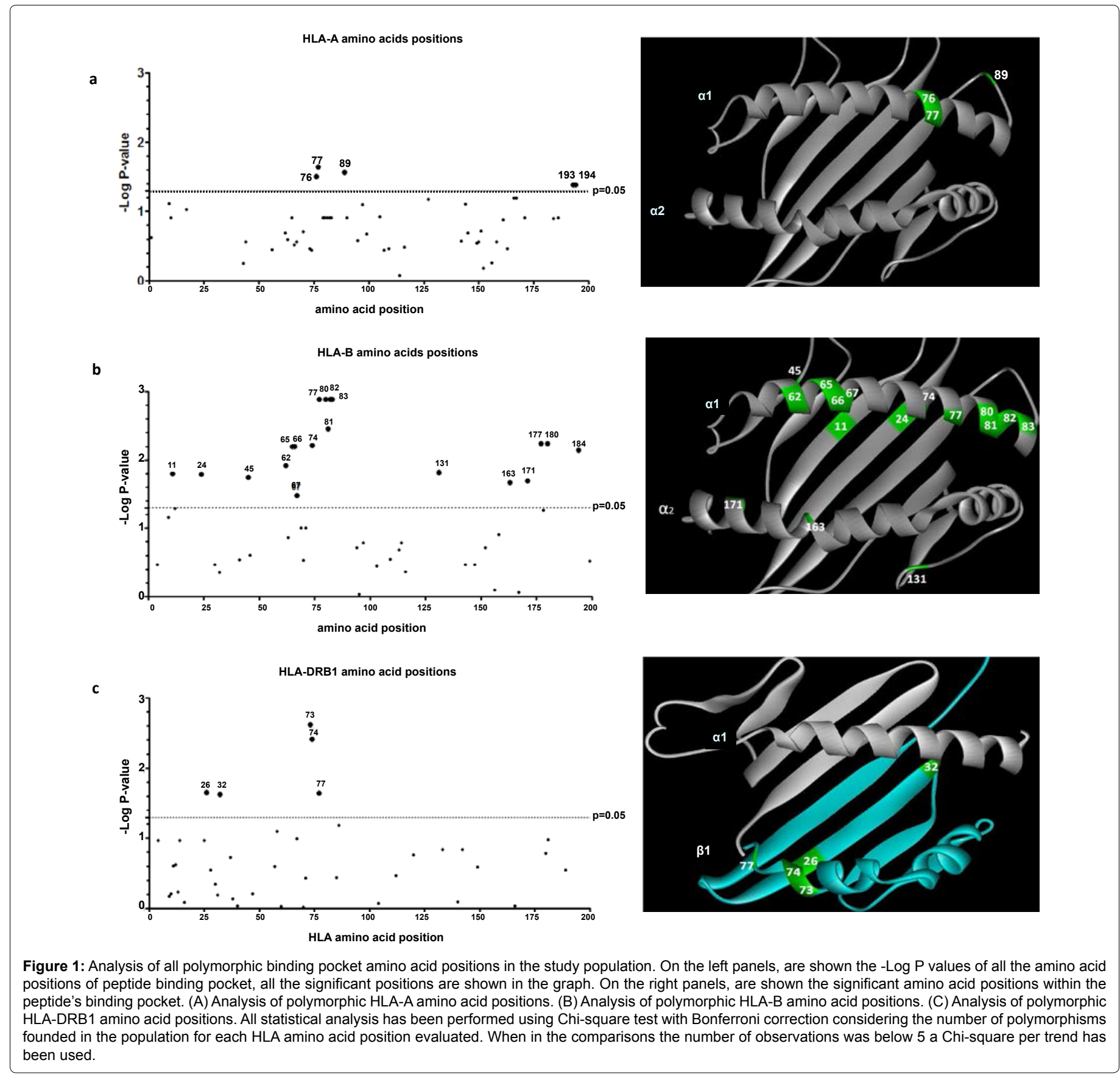


Citation: Montesano C, Bonanno CT, Grifoni A, Di Sano C, Palma P, et al. (2014) Impact of Human Leukocyte Antigen Polymorphisms in Human Immunodeficiency Virus Progression in a Paediatric Cohort Infected with a Mono-phyletic Human Immunodeficiency Virus-1 Strain. J AIDS Clin Res 5: 282. doi:10.4172/2155-6113.1000282

Page 4 of 6

\begin{tabular}{|c|c|c|c|c|c|c|}
\hline & Binding pocket ${ }^{\#}$ & LTNP & Fast progressor & & LTNP vs. Fast progressor & LTNP vs. Fast progressor \\
\hline HLA-A & & $\mathbf{N}$ & $\mathbf{N}$ & 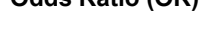 & Uncorrected $p$ value & Bonferroni's corrected $p$ value \\
\hline $62 \mathrm{Q}$ & B & $18(69.23 \%)$ & $8(34.78 \%)$ & 4.2187 & 0.0336 & 0.2881 \\
\hline \multicolumn{7}{|l|}{ HLA-B } \\
\hline $11 S$ & $\mathrm{~F}^{*}$ & $2(7.69 \%)$ & $8(34.78 \%)$ & 0.1562 & 0.0463 & 0.0463 \\
\hline $24 \mathrm{~A}$ & B & $21(80.77 \%)$ & $11(47.83 \%)$ & 4.5818 & 0.0343 & 0.1344 \\
\hline $24 S$ & B & $4(15.38 \%)$ & $13(56.52 \%)$ & 0.1398 & 0.0066 & 0.0546 \\
\hline $45 \mathrm{E}$ & B & $4(15.38 \%)$ & $13(56.52 \%)$ & 0.1398 & 0.0066 & 0.1165 \\
\hline $45 \mathrm{~T}$ & B & $20(76.92 \%)$ & $8(34.78 \%)$ & 6.25 & 0.0072 & 0.2921 \\
\hline $62 \mathrm{G}$ & $A$ and $B$ & $10(38.46 \%)$ & $2(8.7 \%)$ & 6.5625 & 0.0370 & 0.351 \\
\hline $65 R$ & $A$ and $B$ & $11(42.31 \%)$ & $2(8.7 \%)$ & 7.7 & 0.0195 & 0.6277 \\
\hline $66 \mathrm{~N}$ & B & $11(42.31 \%)$ & $2(8.7 \%)$ & 7.7 & 0.0195 & 0.0195 \\
\hline $67 \mathrm{M}$ & B & $11(42.31 \%)$ & $2(8.7 \%)$ & 7.7 & 0.0195 & 0.2429 \\
\hline $70 \mathrm{~S}$ & B & $11(42.31 \%)$ & $2(8.7 \%)$ & 7.7 & 0.0195 & 0.1775 \\
\hline $74 \mathrm{D}$ & $\mathrm{F}$ & $4(15.38 \%)$ & $12(52.17 \%)$ & 0.1667 & 0.0149 & 0.0149 \\
\hline $77 \mathrm{~N}$ & $\mathrm{~F}$ & $22(84.61 \%)$ & $11(47.83 \%)$ & 6 & 0.0149 & 0.085 \\
\hline 801 & $\mathrm{~F}$ & $21(80.77 \%)$ & $8(34.78 \%)$ & 7.875 & 0.0029 & 0.0353 \\
\hline $81 \mathrm{~A}$ & $\mathrm{~F}$ & $22(84.61 \%)$ & $11(47.83 \%)$ & 6 & 0.0149 & 0.0149 \\
\hline $82 \mathrm{~L}$ & External§ & $23(88.46 \%)$ & $11(47.83 \%)$ & 8.3636 & 0.0056 & 0.0056 \\
\hline $83 R$ & Externals & $23(88.46 \%)$ & $11(47.83 \%)$ & 8.3636 & 0.0056 & 0.0056 \\
\hline $94 \mathrm{~T}$ & $\mathrm{~F}$ & $13(50 \%)$ & $22(91.3 \%)$ & 0.0454 & 0.0013 & 0.0013 \\
\hline $95 \mathrm{~L}$ & $\mathrm{~F}$ & $6(23.08 \%)$ & $13(56.52 \%)$ & 0.2307 & 0.0354 & 0.2244 \\
\hline $97 \mathrm{~S}$ & $\mathrm{~F}$ & $5(19.23 \%)$ & $14(60.87 \%)$ & 0.153 & 0.0071 & 0.1783 \\
\hline \multicolumn{7}{|l|}{ HLA-DR } \\
\hline $26 Y$ & 4 & $2(7.69 \%)$ & $8(34.78 \%)$ & 2.380952381 & 0.0463 & 0.912684596 \\
\hline $73 \mathrm{~A}$ & 4 & $26(100 \%)$ & $18(78.26 \%)$ & N.A. & 0.0417 & 0.0417 \\
\hline $73 G$ & 4 & $9(34.62 \%)$ & $16(69.56 \%)$ & 0.231617647 & 0.0311 & 0.0311 \\
\hline $74 R$ & 4 & $2(7.69 \%)$ & $8(34.78 \%)$ & 0.15625 & 0.0463 & 0.372758417 \\
\hline $77 \mathrm{~N}$ & 4 & $2(7.69 \%)$ & $8(34.78 \%)$ & 0.15625 & 0.0463 & 0.158747122 \\
\hline $77 \mathrm{~T}$ & 4 & $26(100 \%)$ & $18(78.26 \%)$ & N.A. & 0.0417 & 0.149950893 \\
\hline
\end{tabular}

Table 2: HLA phenotypic analysis of binding pocket amino acid polymorphism significantly associated with the study groups.

80( $(\mathrm{P}=0.013), 81(\mathrm{P}=0.003)$ resulted associated either with LTNP or FP (Figure 1B).

Further it has been evaluated the 114 amino acid polymorphisms in the HLA-B locus (see S3).

Considering B-pocket, amino acid polymorphism $66 \mathrm{~N}$ resulted significantly associated with LTNP after Bonferroni's correction $(\mathrm{LTNP}=42.31 \% \mathrm{FP}=8.7 \% \mathrm{OR}=7.7 ; \mathrm{p}<0.02$, Table 2$)$.

In the context of F-pocket, amino acid polymorphisms $11 \mathrm{~S}$ $(\mathrm{LTNP}=7.69 \% \mathrm{FP}=34.78 \%$ OR=0.156 $\mathrm{P}<0.05), 74 \mathrm{D} \quad(\mathrm{LTNP}=15.38 \%$, $\mathrm{FP}=52.17 \%, \mathrm{OR}=0.167 ; \mathrm{p}<0.015)$ and $94 \mathrm{~T}(\mathrm{LTNP}=15.38 \%, \mathrm{FP}=52.17 \%$, $\mathrm{OR}=0.045 ; \mathrm{p}<0.001)$ have been found associated with FP after Bonferroni's correction (Table 2).

Further, the analysis has been focused to another pattern of amino acid positions $(80,81,82$ and 83$)$ located in or in the proximity of the F-pocket and belonging to $\alpha_{1}$ (Figure $1 \mathrm{~B}$, right panel).

In particular, 80I $(\mathrm{LTNP}=80.77 \%, \mathrm{FP}=34.78 \%, \mathrm{OR}=7.86 ; \mathrm{p}<0.036)$, $81 \mathrm{~A}(\mathrm{LTNP}=84.61 \%, \mathrm{FP}=47.83 \%, \mathrm{OR}=6 ; \mathrm{p}<0.015), 82 \mathrm{~L}(\mathrm{LTNP}=88.46 \%$, $\mathrm{FP}=47.83 \%, \mathrm{OR}=7.86 ; \mathrm{p}<0.006)$ and $83 \mathrm{R}(\mathrm{LTNP}=88.46 \%, \mathrm{FP}=47.83 \%$, $\mathrm{OR}=7.86 ; \mathrm{p}<0.006$ ) have been found significantly associated with LTNP after Bonferroni's correction (Figure 1B and Table 2).

Considering that these four amino acid polymorphisms belong to epitope Bw4, previously found associated with HIV delayed disease progression [16-18], all the HLA-B alleles have been classified accordingly to carry Bw4 (77N, 80I/T, 81A, 82L, 83R) or its counterpart Bw6 (77S, 80N, 81L, 82R, 83G) epitope to performe a genotypic analysis.

As for the analysis of the polymorphisms, the phenotype frequency of Bw4 epitope resulted associated with LTNP (LTNP $=88.46 \%$, $\mathrm{FP}=47.83 \%, \mathrm{OR}=8.36 ; \mathrm{p}<0.006$ ). To further deep the analysis, genomic distribution of Bw4 and Bw6 epitopes in the study groups has been evaluated (Figure 2). An overall significantly different distribution of the homozygous and heterozygous for Bw4 and Bw6 in the study groups has been found $(\mathrm{p}<0.002)$, with $\mathrm{Bw} 4$ and Bw6 homozygosis resulted significantly increased in LTNP and FP, respectively (Figure 2). No differences in the heterozygous Bw4/Bw6 frequency in LTNP vs FP have been observed (Figure 2).

Finally, the study of the 44 amino acid polymorphic positions in HLA-DR locus showed amino acid positions $26(\mathrm{P}=0.022), 32$ $(\mathrm{P}=0.024), 73(\mathrm{P}=0.002), 74(\mathrm{P}=0.004)$ and $77(\mathrm{P}=0.023)$ significant after Bonferroni's correction (Figure 1C).

The analysis of the 114 amino acid polymorphisms in HLA-DR showed only for amino acid position A73 a significant association with LTNP (LTNP $=100 \%, F P=78.26 \%$, OR=N.A.; $<<0.042)$ and for $\mathrm{G} 73$ with $\mathrm{FP}(\mathrm{LTNP}=34.62 \%, \mathrm{FP}=69.56 \%, \mathrm{OR}=0.23$; $\mathrm{p}<0.032)$ after Bonferroni's correction (Table 2 and S3).

\section{Discussion}

HIV-specific T-cell response, and in particular CTL, plays a key role 


\section{HLA-B Bw4/Bw6 epitope}

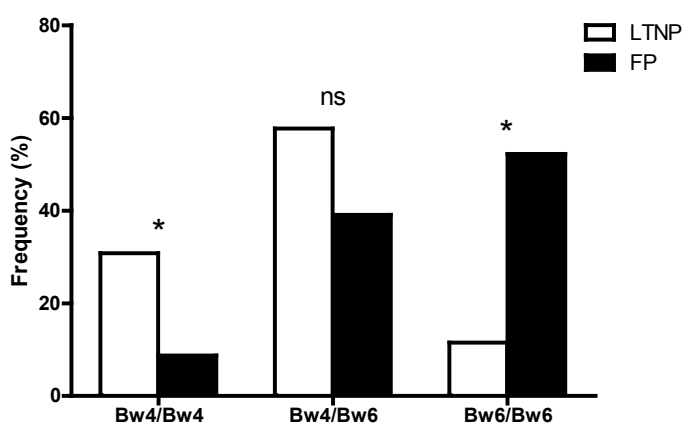

Figure 2: Genotype frequencies of HLA-B Bw4 and Bw6 epitopes in the study groups. Frequency of Bw4/Bw4 homozygous, Bw4/Bw6 heterozygous and Bw6/Bw6 homozygous is reported as percentage for LTNP (black bars) and FP (open bars). Statistical analysis between groups has been performed with chi-square and chi-square per trend test as appropriate. $" p<0.05$; ns=not significant.

in controlling HIV infection $[1,19]$. As the T-cell response is dictated by HLA molecules, the individual's variation in the HLA class I and II alleles has a profound effect on the outcome of infection and disease progression to AIDS [2,19].

In this study, in a defined cohort of children infected during an hospital outbreak with a monophyletic strain of HIV-1 [9], the role of amino acid polymorphisms determining specific characteristics of the HLA peptide binding pocket have been defined. In agreement with previous observations, but with the limitation of the small study population studied, the data indicate that the ability of certain HLA peptide binding pockets to present a specific set of epitopes against which the subject can mount an immune response, might represent the basis of the susceptibility to AIDS progression, at least for the HLA-B locus $[1,2,6,19]$.

Only limited studies have been focused on the HLA class I and II amino acid polymorphisms that might play a role in the disease progression to AIDS. In a recent large multinational study focused on the model of HIV controllers [6], one polymorphic position of HLA-A (position 77) and three polymorphic positions of HLA-B (67, 70 and 97) have been found to play a major role in controlling HIV infection. With limit of the small study population and the different study design, but the strong advantage of the minimal HIV strain variability and other genetic background, this study is in agreement with these observations (see also Figure 1B).

Specifically, 67M and 70S resulted associated with HIV controllers and 97S associated with progressors in Pereyra et al. 2010. In this study the same associations are observed even if the significant $\mathrm{P}$ value is loss after Bonferroni's correction, due to the small number of patient analyzed in this study (see Supplementary information S3).

As expected from previous immunogenetic studies, the large number of residues found associated with LTNP or progression to AIDS, have been located in the HLA-B locus $[2,6,20]$, particularly in the primary anchor binding B-pocket and F-pocket of HLA-B (see also Table 2).

This study shows a strong contribution of the F-pocket of HLA-B in HIV disease progression and non progression. Specifically, two patterns of polymorphism have been identified. A first pattern includes polymorphic variants $80 \mathrm{I}, 81 \mathrm{~A}, 82 \mathrm{~L}, 83 \mathrm{R}$ associated with LTNP, while a second pattern includes $74 \mathrm{D}$ and $94 \mathrm{~T}$ polymorphic variants associated with progression to AIDS.

The polymorphic variants $74 \mathrm{D}$ and $94 \mathrm{~T}$ are involved in positions located in F-pocket playing a minor role in peptide binding [21]. However, when these residues are present, they are associated with the binding of hydrophobic amino acid variants $[14,22]$.

On the opposite, residues $80,81,82$ and 83 are playing a primary key role in the interaction with the peptide in F-pocket of HLA-B [21]. The LTNP associated pattern $80 \mathrm{I}, 81 \mathrm{~A}, 82 \mathrm{R}, 83 \mathrm{~L}$ is typical of HLA-B alleles falling in supertypes such as B58 (grouping most of the HLA-B ${ }^{\star} 57$ and HLA-B ${ }^{\star} 58$ alleles) and B27, already found associated with slow progression to AIDS [2].

The same amino acid positions are involved in the epitope Bw4 and Bw6. When HLA-B alleles are classified according with carrying Bw4/ Bw6 epitope, a strong contribution of Bw4 in delaying HIV progression has been observed. These results are in agreement with previous association of Bw4 homozygous and the control of HIV viremia [18].

The importance of epitope Bw4 is not only in its direct interaction with the bound peptide which therefore modify the CD8+T cell recognition. In fact, it is also a ligand for KIR3DL1, an NK's inhibitory receptor [23,24], suggesting a strong contribution of the innate immune response in controlling HIV progression and confirming the key role played by HLA-B molecules [16].

Other amino acid polymorphic positions of HLA class I molecule, might play an additional role in HIV progression upon interacting with NK receptors. Among them, amino acid position 194 located in HLA-Ba domain might contribute to HIV progression (see also Figure 1). This polymorphic position not only influence the KIR3DL1 interaction [25], but represents also the binding site for LILRB1, another NK's receptor [26]. In this context, we have recently reported an immunoinformatic approach assessing the contribution of the different amino acid polymorphisms at position 194 of HLA-B in the interaction with LILRB1 and their role in HIV progression [27].

HLA-DR polymorphisms presented a lower impact in HIV progression, as already underlined in previous studies [6,28]. Only polymorphic residues in pocket 4 , determining the peptide bound allele specificity and repertoire of antigenic peptides presented by HLA-DR molecules $[13,29]$, seems to play a role in HIV progression. However, the specific contribution of Ala/Gly 73 polymorphisms to peptide binding capability is difficult to assess.

Altogether, these observations suggest that the progression to AIDS might be in part determined by the binding capability of B-pocket and F-pocket of HLA-B and in part by the interaction between HLA-B molecules and NK's inhibithory receptor which therefore regulate also the innate immune response.

In conclusion, with the limit of the small study population but the strong advantage of the homogeneous ethnic background and monophyletic HIV-1 strain infection in the evaluated cohort, the observations here reported might have important implications for a better control of the disease. The extension of this study by using immunoinformatic tools for the prediction of T-cells epitopes to the entire HIV-1 genome products, might shed new light on the mechanisms behind the association of genetic susceptibility to HLA in HIV-1 and represent a powerful tool for a more effective strategy in vaccine and diagnostics design ensuring wider coverage of the populations, including genetically susceptible subjects. 
Citation: Montesano C, Bonanno CT, Grifoni A, Di Sano C, Palma P, et al. (2014) Impact of Human Leukocyte Antigen Polymorphisms in Human Immunodeficiency Virus Progression in a Paediatric Cohort Infected with a Mono-phyletic Human Immunodeficiency Virus-1 Strain. J AIDS Clin Res 5: 282. doi:10.4172/2155-6113.1000282

Page 6 of 6

\section{Acknowledgements}

We would like to thank Marco Pallante (University of Tor Vergata, Roma, IT) for the assistance with the immunobioinformatic analysis and the helpful discussion. This study has been supported in part by a project of the University of Rome "Tor Vergata" (ex 60\% MIUR, 2006) to MA.

We wish to thank to Prof. Graham Lord (Guy's and St Thomas' Hospitals and King's College London, UK) for the critical reading and editing of the manuscript.

\section{References}

1. Kaur G, Mehra N (2009) Genetic determinants of HIV-1 infection and progression to AIDS: immune response genes. Tissue Antigens 74: 373-385.

2. Trachtenberg EA, Erlich HA (2001) A Review of the Role of the Human Leukocyte Antigen (HLA) System as a Host Immunogenetic Factor Influencing HIV Transmission and Progression to AIDS. In: Korber BT, Brander C, Haynes BF, Koup R, Kuiken C, et al. (eds.) HIV Molecular Immunology 2001. Theoretical Biology and Biophysics Group, Los Alamos National Laboratory, Los Alamos, NM, LA-UR, 43-60.

3. Huang X, Ling H, Mao W, Ding X, Zhou Q, et al. (2009) Association of HLA-A $\mathrm{B}, \mathrm{DRB} 1$ alleles and haplotypes with HIV-1 infection in Chongqing, China. BMC Infect Dis 9: 201

4. O'Brien SJ, Gao X, Carrington M (2001) HLA and AIDS: a cautionary tale. Trends Mol Med 7: 379-381.

5. Kiepiela P, Leslie AJ, Honeyborne I, Ramduth D, Thobakgale C, et al. (2004) Dominant influence of HLA-B in mediating the potential co-evolution of HIV and HLA. Nature 432: 769-775.

6. International HIV Controllers Study, Pereyra F, Jia X, McLaren PJ, Telenti A, et al. (2010) The major genetic determinants of HIV-1 control affect HLA class I peptide presentation. Science 330: 1551-1557.

7. Stewart-Jones GB, Simpson P, van der Merwe PA, Easterbrook P, McMichael AJ, et al. (2012) Structural features underlying T-cell receptor sensitivity to concealed MHC class I micropolymorphisms. Proc Natl Acad Sci U S A 109 : E3483-3492.

8. Gregersen PK, Silver J, Winchester RJ (1987) The shared epitope hypothesis. An approach to understanding the molecular genetics of susceptibility to rheumatoid arthritis. Arthritis Rheum 30: 1205-1213.

9. de Oliveira T, Pybus OG, Rambaut A, Salemi M, Cassol S, et al. (2006) Molecular epidemiology: HIV-1 and HCV sequences from Libyan outbreak. Nature 444: 836-837.

10. Galgani A, Mancino G, Martínez-Labarga C, Cicconi R, Mattei M, et al. (2013) HLA-A, -B and -DRB1 allele frequencies in Cyrenaica population (Libya) and genetic relationships with other populations. Hum Immunol 74: 52-59.

11. Yerly S, Quadri R, Negro F, Barbe KP, Cheseaux JJ, et al. (2001) Nosocomia outbreak of multiple bloodborne viral infections. J Infect Dis 184: 369-372.

12. CDC (1994) Revised classification system for human immunodeficency virus infection in children less than 13 years of age. MMWR Morb Mortal Wkly Rep 43: $1-10$.

13. Lund O, Nielsen M, Kesmir C, Petersen AG, Lundegaard C, et al. (2004) Definition of supertypes for HLA molecules using clustering of specificity matrices. Immunogenetics 55: 797-810.

14. Sidney J, Peters B, Frahm N, Brander C, Sette A (2008) HLA class I supertypes: a revised and updated classification. BMC Immunol 9: 1.
15. Voorter CE, Amicosante M, Berretta F, Groeneveld L, Drent M, et al. (2007) HLA class II amino acid epitopes as susceptibility markers of sarcoidosis. Tissue Antigens 70: 18-27.

16. Carrington M, Alter G (2012) Innate immune control of HIV. Cold Spring Harb Perspect Med 2: a007070.

17. Anfossi N, André P, Guia S, Falk CS, Roetynck S, et al. (2006) Human NK cell education by inhibitory receptors for MHC class I. Immunity 25: 331-342.

18. Flores-Villanueva PO, Yunis EJ, Delgado JC, Vittinghoff E, Buchbinder S, et al (2001) Control of HIV-1 viremia and protection from AIDS are associated with HLA-Bw4 homozygosity. Proc Natl Acad Sci U S A 98: 5140-5145.

19. McMichael AJ, Rowland-Jones SL (2001) Cellular immune responses to HIV. Nature 410: 980-987.

20. Hertz T, Nolan D, James I, John M, Gaudieri S, et al. (2011) Mapping the landscape of host-pathogen coevolution: HLA class I binding and its relationship with evolutionary conservation in human and viral proteins. J Virol 85: 13101321.

21. Madden DR (1995) The three-dimensional structure of peptide-MHC complexes. Annu Rev Immunol 13: 587-622.

22. Bade-Doeding C, DeLuca DS, Seltsam A, Blasczyk R, Eiz-Vesper B (2007) Amino acid 95 causes strong alteration of peptide position Pomega in HLA-B*41 variants. Immunogenetics 59: 253-259.

23. Thananchai H, Gillespie G, Martin MP, Bashirova A, Yawata N, et al. (2007) Cutting Edge: Allele-specific and peptide-dependent interactions between KIR3DL1 and HLA-A and HLA-B. J Immunol 178: 33-37.

24. Vivian JP, Duncan RC, Berry R, O'Connor GM, Reid HH, et al. (2011) Killer cel immunoglobulin-like receptor 3DL1-mediated recognition of human leukocyte antigen B. Nature 479: 401-405.

25. Sanjanwala B, Draghi M, Norman PJ, Guethlein LA, Parham P (2008) Polymorphic sites away from the Bw4 epitope that affect interaction of Bw4+ HLA-B with KIR3DL1. J Immunol 181: 6293-6300.

26. Jones DC, Kosmoliaptsis V, Apps R, Lapaque N, Smith I, et al. (2011) HLA class I allelic sequence and conformation regulate leukocyte Ig-like receptor binding. J Immunol 186: 2990-2997.

27. Grifoni A, Montesano C, Palma P, Salerno A, Colizzi V, et al. (2013) Role of HLA-B Ît-3 domain amino acid position 194 in HIV disease progression. Mol Immunol 53: 410-413.

28. Carrington M, Walker BD (2012) Immunogenetics of spontaneous control of HIV. Annu Rev Med 63: 131-145.

29. Sturniolo T, Bono E, Ding J, Raddrizzani L, Tuereci O, et al. (1999) Generation of tissue-specific and promiscuous HLA ligand databases using DNA microarrays and virtual HLA class II matrices. Nat Biotechnol 17: 555-561. 